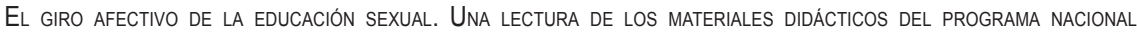
de Educación Sexual Integral. Páginas 65-80 en Revista de la Escuela de Ciencias de la Educación, año 10, NÚMERO 9, ENERO A DICIEMBRE DE 2014. ISSN 1851-6297. ISSN EN LÍNEA 2362-3349.

\title{
EL GIRO AFECTIVO DE LA EDUCACIÓN SEXUAL. UNA LECTURA DE LOS MATERIALES DIDÁCTICOS DEL PROGRAMA NACIONAL DE EDUCACIÓN SEXUAL INTEGRAL
}

\author{
Por Facundo Gustavo Boccardi (Universidad Nacional de Córdoba)*, Argentina. \\ facundoccardi@gmail.com
}

Recibido: 30/06/2014 Aceptado: 25/10/2014

\section{Resumen}

El privilegio de la afectividad constituye una de las operaciones centrales emprendida por el actual proceso de la Ley Nacional de Educación Sexual Integral. Con ello, se pretende descentrar el foco históricamente ubicado sobre las vicisitudes biomédicas de la población escolar en edad reproductora. Teniendo en cuenta un marco de referencia más amplio denominado "giro afectivo", el siguiente artículo realiza una lectura de la construcción discursiva de la afectividad en los materiales didácticos producidos por el Programa Nacional de Educación Sexual Integral con el objeto de demarcar este nuevo territorio educativo.

\section{Palabras Clave}

Educación Sexual Integral - Afectividad - Giro afectivo - Sexualidad - Semiótica.

\begin{abstract}
The privilege of affectivity is one of the core operations undertaken by the current process of national law Integral Sexual Education. This is intended to decentralize the focus historically located on biomedical vicissitudes of the school population in reproductive age. Considering a broader frame of reference called "affective turn", the following article takes a reading of the discursive construction of emotion in teaching materials produced by the National Comprehensive Sex Education Program in order to delineate this new educational territory.
\end{abstract}

* Licenciado en Letras modernas en la Facultad de Filosofía y Humanidades de la Universidad Nacional de Córdoba (UNC). Participa desde el 2007 en el Programa de Estudios de Género radicado en el CEA - UNC. 
Revista de la Escuela de Ciencias de la Edducación, año 10, número 9, enero a diciembre de 2014. PÁginas 65-80. ISSN 1851-6297. ISSN EN LÍNEA 2362-3349. EL GIRO AFECTIVO DE LA EDUCACIÓN SEXUAL. UNA LECTURA DE LOS MATERIALES didÁcticos del programa nacional de Educación SeXual Integral. Facundo Gustavo Boccardi.

\section{Key Words}

Comprehensive sex education - Affectivity - Affective turn - Sexuality - Semiotic.

\section{Introducción}

"The affective turn" es el último giro que ha bautizado la academia anglosajona. Con cada vez mayor pregnancia en los circuitos editoriales y en la agenda académica, esta etiqueta da cuenta de la proliferación creciente del abordaje de los afectos y las emociones en el campo investigativo de las ciencias sociales y humanas en las últimas décadas. Esta tendencia de la academia pone de relieve la progresiva importancia que adquieren las dimensiones afectivas y emocionales en la vida pública. De este modo, algunos autores nombran las sociedades contemporáneas como "sociedades afectivas" (Cfr. Squire, 2001) y existe consenso en la percepción de transformaciones que pueden ser enmarcadas en un proceso de emocionalización de la esfera pública (Cfr. Greco y Stenner, 2008; Gregg y Seigworth, 2010).

En esta instancia, no nos detendremos a evaluar ni la novedad ni el alcance explicativo de este giro, sino que aprovecharemos su impulso para ajustar el foco de la indagación en una zona específica de la Educación Sexual Integral argentina. La combinación de los términos "afectividad" y "educación" forma parte, sin dudas, de la agenda local tanto del campo investigativo como de las políticas públicas. En el campo de la educación sexual, la afectividad aparece como una de las claves que permite dislocar el centro situado históricamente en la prevención de infecciones de transmisión sexual y embarazos adolescentes (Cfr. Boccardi, 2013). El presente artículo recorre los materiales didácticos oficiales destinados al abordaje de la temática en los niveles inicial, primario y secundario del sistema educativo con el objeto de relevar el funcionamiento de la tópica afectiva en el entramado discursivo de la educación sexual. La tematización de los afectos en estos materiales se lleva adelante mediante un conjunto de operaciones discursivas que establecen relaciones de proximidad y distanciamiento con otros tópicos demarcando un territorio propio de creciente relevancia. En esta oportunidad, nuestro interés se limita a la descripción de estas operaciones mediante herramientas analíticas de la semiótica con el objeto de ofrecer insumos para la comprensión de la zona de la afectividad en los discursos de la educación sexual provenientes de los aparatos del Estado.

\section{Aproximación a las condiciones históricas de emergencia de la Educación Sexual argentina}

Consideramos que la década del sesenta puede ser tomada como un punto de partida para pensar los procesos históricos que se condensan con diferentes tensiones y paradojas en el paisaje contemporáneo de la educación 
Revista de la Escuela de Ciencias de la Educación, año 10, número 9, enero a diciembre de 2014. Páginas 65-80. ISSN 1851-6297. ISSN EN LINEA 2362-3349. EL GIRO AFECTIVO DE LA EDUCACIÓN SEXUAL. UNA LECTURA DE LOS MATERIALES didÁcticos del programa nacional de Educación Sexual Integral. Facundo Gustavo Boccardi.

sexual. Esta década introduce una serie de desplazamientos en el terreno de la sexualidad que han sido aglutinados bajo el nombre de "revolución sexual". En este horizonte, marcado por los cambios en los modelos familiares, la mayor visibilidad de las reivindicaciones de movimientos feministas y las nuevas orientaciones para la crianza de los niños, la educación sexual adquiere relevancia en la agenda de las políticas educativas en distintos países de Europa, Estados Unidos y América Latina. La educación sexual era considerada por gran parte de los países una necesidad que debía ser cubierta por las escuelas (Cfr. Wainerman, Di Virgilio y Chami, 2008). También, en esta década, la difusión del uso de anticonceptivos y la expansión del discurso sobre el derecho al placer operaron como factores que potenciaron el aumento de estudios sobre la sexualidad en el campo de las ciencias sociales (Gogna, 2005; Cosse, 2010). En nuestro país, las primeras experiencias en el campo de la educación sexual se ubican en este período histórico (Cfr. Felitti, 2009) atravesado por procesos embrionarios de redefinición de los roles rígidos que determinaban las configuraciones familiares cuyos efectos implicaron la liberalización de algunos mandatos de género y sexualidad (Esquivel, 2013).

En los años ochenta, con la emergencia del VIH-Sida, se multiplicó el debate sobre la educación sexual, considerándolo una vía para combatir la epidemia. Es así que en las décadas de los ochentas y noventas, varios países de América Latina aprobaron leyes que incorporaban la educación sexual en el currículum de la educación formal. En Argentina, sin embargo, los gobiernos dictatoriales impidieron el desarrollo de políticas públicas que avanzaran en esa dirección, afianzando una fórmula rígida estructurada por preceptos morales sexuales arraigados en el catolicismo que se traducían en políticas natalistas. Recién con el advenimiento de la democracia en 1983, se inició un período de definición de políticas de salud y derechos sexuales y reproductivos.

La década de los noventas introdujo transformaciones significativas en el campo legislativo que funcionaron como condiciones de posibilidad para las políticas contemporáneas. En este sentido, existe consenso en considerar que la reforma constitucional de 1994 constituyó un hito en este proceso dado que abrió un espacio de interlocución para que el movimiento de mujeres planteara demandas que permanecían postergadas (Birgin, 1996). Este marco le otorga estatus constitucional a tratados internacionales, declaraciones y convenciones que dan surgimiento a una nueva categoría de derechos o ratifican los ya existentes, reconociendo a los niños, niñas y adolescentes como sujetos de derecho.

En el año 2002, con la creación del Programa de Salud Sexual y Procreación Responsable (Ley Nacional 25.673), se propone que la población alcance el nivel más elevado de salud sexual y procreación responsable, garantizando el derecho a la información y a la educación sexual como responsabilidad del Estado, convocando la participación del Ministerio de Educación para hacerlos efectivos. 
Revista de la Escuela de Ciencias de la Educación, año 10, número 9, enero a diciembre de 2014. Páginas 65-80. ISSN 1851-6297. ISSN EN LINEA 2362-3349. EL GIRO AFECTIVO DE LA EDUCACIÓN SEXUAL. UNA LECTURA DE LOS MATERIALES didÁcticos del programa nacional de EduCación Sexual Integral. Facundo Gustavo Boccardi.

Así, luego de un extenso debate, en octubre de 2006, se sanciona la ley que crea el Programa Nacional de Educación Sexual Integral (Ley 26.150). Con esta operación jurídica, se establece la obligatoriedad de impartir educación sexual, en todos los niveles y en todas las modalidades del sistema educativo público de gestión estatal y de gestión privada y en todas las carreras de formación docente del país. En los debates legislativos precedentes a la sanción de la Ley Nacional 26.150 que ocuparon la arena mediática, uno de los núcleos problemáticos versó acerca de la posibilidad del dispositivo pedagógico de incorporar la sexualidad como contenido a ser impartido. El problema residía justamente en la relación de los sujetos que componen la relación pedagógica con el conocimiento y la ignorancia: el sujeto que debía saber no sabía y el sujeto que debía ignorar no ignoraba. La sexualidad, así, ponía en jaque por un momento esta dicotomía estructurante del discurso pedagógico (Cfr. Boccardi, 2008). Luego prevaleció la combinación del peso de "los problemas de la sexualidad adolescente" con el peso de la escuela como espacio legítimo para garantizar el acceso a derechos.

Casi dos años más tarde, en abril de 2008, el Consejo Federal de Educación publica los "Lineamientos curriculares para la Educación Sexual Integral" que definen el piso común de contenidos curriculares válidos para todos los niveles del sistema educativo. El lapso que separa estos dos acontecimientos estuvo signado por la disputa acerca de la definición de los contenidos de la Educación Sexual Integral. Ni bien es sancionada la ley, el Ministerio de Educación de la Nación designa una "comisión de expertos" con el objetivo de redactar un documento que sirviera de insumo para la producción definitiva de los lineamientos por parte del Consejo Federal de Educación. Esta comisión conformada por intelectuales reconocidas en el campo educativo con trayectoria en el área de la educación sexual (1) y representantes de tres instituciones religiosas (la católica, la evangélica y la judía) (2) trabajó durante el año 2007 sin llegar a un acuerdo unánime, ya que las representantes de la iglesia católica se negaron a "suscribir un documento que hiciera mención a la perspectiva de género y que silenciara el rol de los padres en la educación sexual de sus hijos" (Esquivel, 2013 p. 101). Debido a la ausencia de una propuesta consensuada, finalmente se elaboraron dos dictámenes - uno de mayoría y otro de minoría- que operaron como insumos para el proceso de redacción final de los lineamientos curriculares llevado a cabo por técnicos de los ministerios de educación de las provincias en el marco del Consejo Federal de Educación.

Desde el año 2009 hasta finales del 2012, el Programa Nacional de Educación Sexual integral produce materiales didácticos destinados a abordar los contenidos establecidos por los lineamientos curriculares para los niveles inicial, primario y secundario. Estos materiales se encuentran compuestos por folletería, cortos audiovisuales, láminas y la Serie Cuadernos de ESI (3) compuesta por cuatro cuadernos diferenciados por nivel educativo. La Serie Cuadernos de ESI 
Revista de la Escuela de Ciencias de la Educación, año 10, número 9, enero a diciembre de 2014. Páginas 65-80. ISSN 1851-6297. ISSN EN LINEA 2362-3349. EL GIRO AFECTIVO DE LA EDUCACIÓN SEXUAL. UNA LECTURA DE LOS MATERIALES didÁcticos del programa nacional de Educación Sexual Integral. Facundo Gustavo Boccardi.

destinada a los y las docentes del sistema educativo presenta una selección de contenidos de los lineamientos curriculares, articula fundamentaciones conceptuales y ofrece, principalmente, actividades didácticas para implementar en las aulas. La producción y distribución masiva de estos materiales en todas las escuelas del país responde a una estrategia del Programa Nacional de Educación Sexual Integral tendiente a la sensibilización y capacitación docente y, finalmente, a la concreción definitiva de una política pública de educación sexual a nivel nacional.

\section{Acerca de los materiales didácticos}

\subsection{Estructura de la Serie Cuadernos de ESI}

Los materiales didácticos que abordamos componen la Serie Cuadernos de ESI. Se trata de un dispositivo pedagógico producido por el Programa Nacional de Educación Sexual Integral con el objetivo de efectivizar la implementación de la Ley 26.150 en todas las instituciones educativas de la República Argentina. Producidos a partir de la publicación de los Lineamientos curriculares para la Educación Sexual Integral por parte del Consejo Federal de Educación, estos materiales apuntan a proveer de herramientas didácticas a los y las docentes de los niveles inicial, primario y secundario del sistema educativo argentino.

La serie se encuentra compuesta por cuatro cuadernos: uno destinado al nivel inicial, uno al nivel primario y dos destinados al nivel secundario (4). Todos los cuadernos de la serie presentan una estructura similar, en las primeras páginas se ubican las cartas y el prólogo destinados a la presentación y contextualización de la propuesta, luego una "presentación general" que funciona como fundamentación conceptual de la Educación Sexual Integral estableciendo una serie de posicionamientos postulados como centrales (5), finalmente se abordan los contenidos cuya estructura presenta algunas variaciones por nivel.

La sección destinada a los "contenidos y propuestas para el aula" presenta la misma estructura en los cuadernos correspondientes a nivel inicial y primario. En estos casos, los contenidos no se encuentran distribuidos disciplinarmente sino por secciones temáticas que articulan varias disciplinas. Cada una de estas secciones presenta un capítulo titulado "¿Qué y cómo enseñamos? Contenidos de Educación Sexual Integral" donde se explicitan los contenidos expresados en los Lineamientos curriculares para la educación sexual integral correspondiente a cada sección. En el caso de los cuadernos correspondientes al nivel secundario, la presentación de los contenidos se encuentra dispuesta en dos secciones. Por un lado, la sección "la Educación Sexual Integral en las áreas curriculares" establece una separación de contenidos y actividades según la grilla de áreas curriculares establecida en los lineamientos curriculares mencionados. Por otro lado, "la Educación Sexual Integral en espacios curriculares específicos" contiene un conjunto de talleres diferenciados temáticamente 
Revista de la Escuela de Ciencias de la Educación, año 10, número 9, enero a diciembre de 2014. Páginas 65-80. ISSN 1851-6297. ISSN EN LINEA 2362-3349. EL GIRO AFECTIVO DE LA EDUCACIÓN SEXUAL. UNA LECTURA DE LOS MATERIALES didÁcticos del programa nacional de EduCación Sexual Integral. Facundo Gustavo Boccardi.

destinados al "abordaje de los contenidos de Educación Sexual Integral en un tiempo y espacio propios y específicos" (Marina, 2010b p. 67).

\subsection{Las formas del discurso del saber}

La Serie Cuadernos de ESI constituye un dispositivo destinado a la transmisión de saberes acerca de la sexualidad. A diferencia de los libros de texto o manuales escolares (Cfr. Tosi, 2008), el destinatario directo que articula la construcción de estos espacios textuales no son los y las alumnas sino los y las docentes. De acuerdo con Chopin (1992), estos materiales caerían bajo la nominación "guías del maestro" definidas como "(...) un instrumento pedagógico y soporte curricular (...)" que condensa la dimensión ideológica y cultural de la sociedad que lo produce (Chopin, 1992).

En coherencia con la perspectiva teórica adoptada, entendemos que, debido a sus objetivos específicos, la configuración de esta materialidad textual se encuentra regida por mecanismos reguladores inherentes a la "voluntad de verdad" que rige la cultura occidental (Cfr. Foucault, 1987). Institucionalizada bajo el régimen de lo escolar, la voluntad de verdad opera mediante un conjunto de prácticas ligadas a la enseñanza tales como la pedagogía, el sistema de libros, la edición y las bibliotecas. De este modo, su funcionamiento se encuentra constitutivamente determinado "por la forma que tiene el saber de ponerse en práctica en una sociedad, en la que es valorado, distribuido, repartido y, en cierta forma, atribuido" (Foucault, 1987: 22). La forma específica de los discursos destinados a la transmisión de saberes se caracteriza por la complejidad, ya que su composición pone en juego necesariamente diferentes discursos de base cargados con las regulaciones específicas propias de su disciplina de origen. En este sentido, estos discursos están definidos por una operación de recontextualización que consiste en la apropiación de saberes provenientes de diferentes disciplinas y su regulación y homogeneización en la configuración de un orden propio (Cfr. Bernstein, 1990).

Nuestro abordaje de la Serie Cuadernos de ESI no se detendrá específicamente en las operaciones de la recontextualización tendientes a rastrear las disciplinas de base, sino que abordaremos estos discursos de transmisión de saberes como mecanismos productores de objetos discursivos cuyo estatuto cognitivo les confiere una forma específica. De acuerdo con Angenot, entendemos que la forma textual de estos materiales puede ser identificada como "forma argumentativa" (Angenot, 1982: 30). Esta forma discursiva recibe, en el planteo de Angenot, el nombre de "discurso entimemático" ya que la noción de "entimema" es concebida como su unidad funcional de base. En este sentido, un entimema es:

(...) todo enunciado, sobre un tema cualquiera, que plantea un juicio, es decir, opera una puesta en relación del fenómeno tratado con un conjunto conceptual que lo integra o que lo determina. Una 
Revista de la Escuela de Ciencias de la Educación, año 10, número 9, enero a diciembre de 2014. Páginas 65-80. ISSN 1851-6297. ISSN EN LINEA 2362-3349. EL GIRO AFECTIVO DE LA EDUCACIÓN SEXUAL. UNA LECTURA DE LOS MATERIALES didÁcticos del programa nacional de Educación Sexual Integral. Facundo Gustavo Boccardi.

relación de este tipo se produce sólo si ella deriva de un principio regulador más general que se encuentra, por lo tanto, presupuesto en su enunciado (Angenot, 1982, p. 31).

Los discursos entimemáticos desarrollan una estrategia general de orden cognitivo mediante mecanismos que establecen relaciones de determinación con proposiciones reguladoras que se encuentran presupuestas en los enunciados. Estas proposiciones son entendidas como "máximas ideológicas" o "lugares comunes" caracterizados por la aceptabilidad general en un horizonte epocal determinado. La articulación de presupuestos subyacentes constituye un mecanismo regulador e inteligibilizador cuyo análisis nos permitirá visibilizar operaciones del régimen de veridicción que rige estos discursos de transmisión de saberes acerca de la sexualidad.

\section{Sexualidad y afectos: Los niveles de la afectividad}

Uno de los pilares de la herencia moderna que ha sostenido la educación es la separación dicotómica y excluyente entre razón y afectos. La repulsión del segundo término propia, quizás, de toda dicotomía experimenta una alteración notable en el proceso de implementación de la Educación Sexual Integral. La Serie Cuadernos de ESI constituye un espacio textual que articula componentes temáticos afectivos con los mecanismos propios del discurso pedagógico. De este modo, mediante criterios de gradación regidos por las lógicas evolutivas de la matriz escolar, la afectividad aparece incorporada como contenido curricular en los diferentes niveles del sistema educativo (6).

\subsection{Nivel Inicial}

La construcción de la afectividad como objeto discursivo inscribe el reconocimiento y la expresión de los afectos en el marco de la educación. La afectividad se presenta como una articulación que incluye emociones, afectos, sentimientos y necesidades (Marina, 2010: 38) cuyo abordaje educativo implica tanto la identificación de variantes desde una perspectiva evolutiva e histórica como componentes prescriptivos para su manifestación.

En el cuaderno correspondiente al nivel inicial, la expresión de afectos aparece vinculada a las nociones de interés y gusto en una dimensión temporal que implica la posibilidad de variaciones. La siguiente consigna ejemplifica esta perspectiva: “¿Cómo expresaban el enojo cuando eran chiquitos o chiquitas y cómo lo expresan ahora?" (Marina, 2010: 26). Estas variaciones aparecen moduladas por la noción crecimiento (7).

(...) podemos conversar sobre las cosas que les gustaban antes y las de ahora que son más grandes; de este modo pueden ir reconociendo que, a medida que van creciendo, van aprendiendo cosas 
Revista de la Escuela de Ciencias de la Edducación, año 10, número 9, enero a diciembre de 2014. PÁginas 65-80. ISSN 1851-6297. ISSN EN LÍNEA 2362-3349. EL GIRO AFECTIVO DE LA EDUCACIÓN SEXUAL. UNA LECTURA DE LOS MATERIALES didÁcticos del programa nacional de Educación SeXual Integral. Facundo Gustavo Boccardi.

nuevas y sus gustos e intereses van cambiando (...) (Marina, 2010a : 26).

(...) los chicos y las chicas se saludan con un beso en la mejilla, mientras que las personas adultas pueden hacerlo con un beso en la boca cuando forman una pareja (Marina, 2010a : 29).

A partir de la lectura de la última cita, se pone en evidencia la relación establecida entre la descripción de los afectos de acuerdo con parámetros de variación asociados al crecimiento y el enunciado deóntico que establecen modos de expresión de afectos diferenciados para "adultos" y "los chicos y las chicas".

La diferencia entre adultos y niños/as es constitutiva del abordaje de los afectos, ya que no se limita a la dimensión prescriptiva mencionada, referida a los modos apropiados de expresar afectos de acuerdo con la edad, sino que se inscribe en una perspectiva más amplia que vincula el lenguaje y la intimidad. En este sentido, las actividades propuestas para trabajar la dimensión de la afectividad formulan como uno de los objetivos centrales que los niños y las niñas logren "(...) expresar las emociones y los sentimientos con palabras claras que permitan a los adultos acompañar a los chicos y las chicas en sus necesidades" (Marina, 2010a : 36). La ubicación de los afectos en la esfera íntima es una de las operaciones discursivas centrales de la construcción de este objeto. En este mecanismo, la puesta en "palabras claras" adquiere un sentido de comunicabilidad y puesta en público que aparecen constituidas como condiciones necesarias para la protección. Para que los adultos puedan acompañar el proceso signado por las necesidades que viven los educandos, es necesario que estos expresen sus afectos en un lenguaje claro. El rasgo de claridad aquí aparece como el efecto buscado de la educación en el campo afectivo con el objeto de volver visible "las necesidades" que no estarían ubicadas al alcance de la vista de los adultos. La claridad, entendida en un sentido visual, de las palabras permitiría volver externo lo más interior de los niños y las niñas: lo íntimo. Mediante esta operación de orden cognitivo se establece la separación entre quién acompaña y quien es acompañado: quien acompaña debe saber y quien es acompañado debe mostrar.

Con respecto al conjunto de actividades propuestas en el cuaderno, la dimensión de la afectividad aparece configurada por una serie de sentimientos cuyos sujetos son siempre los niños y las niñas que aluden al cuidado de sí mismo y de los-as otros-as. En el plano del cuidado de sí mismo o autocuidado, aparece el sentimiento de vergüenza como un elemento negativo que obstaculiza el conocimiento de las "partes íntimas" del cuerpo (Cfr. Marina, 2010a). El sentimiento de culpa, en la misma línea de sentido, aparece asociado al abuso sexual y también se sostiene que debe ser superado en pos de la integridad del niño o la niña. (Cfr. Marina, 2010a). Por otro lado, en este mismo plano aparece 
Revista de la Escuela de Ciencias de la Educación, año 10, número 9, enero a diciembre de 2014. Páginas 65-80. ISSN 1851-6297. ISSN EN LINEA 2362-3349. EL GIRO AFECTIVO DE LA EDUCACIÓN SEXUAL. UNA LECTURA DE LOS MATERIALES didÁcticos del programa nacional de Educación Sexual Integral. Facundo Gustavo Boccardi.

la noción de promoción de la autoestima (Marina, 2010a) como un proceso de valoración positiva de sí mismo centrado en lo corporal y entendido "como factor protector básico a la hora de pensar en la enseñanza de comportamientos de autoprotección" (Marina, 2010a : 46).

En el plano del cuidado de los otros y las otras, la noción con mayor recurrencia es la de respeto (Cfr. Marina, 2010a p. 39). Las actividades presentan componentes deónticos referidos al respeto de la propiedad privada de los compañeros y las compañeras, al respeto de sus deseos y sentimientos evitando avanzar sobre ellos con la propia expresión de afectos, el respeto de la integridad física evitando las interacciones violentas y el respeto de los gustos de cada uno y cada una evitando la discriminación.

La serie de componentes ubicada en el plano de la afectividad formula conexiones entre, por un lado, el conocimiento de sí mismo, su valoración y protección y, por el otro, el respeto de los otros y las otras. En este último caso, la figura del otro reúne rasgos de simetría y, como hemos visto, su respeto consiste en evitar perjudicarlo mediante las propias conductas. En la tematización del cuidado de sí mismo de los niños y las niñas, aparece también la figura del otro. Se trata puntualmente de un otro adulto que tiene como "responsabilidad" el cuidado y acompañamiento pero que puede no cumplirla posibilitando el maltrato o abuso. La noción de responsabilidad que aparece en esta configuración asocia el no cumplimiento de la función otorgada con la culpa: "Decirle al niño o la niña que no es culpable por lo sucedido. Las personas grandes son responsables de cuidar y proteger a las chicas y a los chicos" (Marina, 2010a : 44). De este modo, la figura del otro cuidador se recorta contra la figura del abuso sexual que supone un sujeto abusador que condensa los riesgos y las amenazas contra los que se debe proteger. Los rasgos de este otro amenazante son la pertenencia al círculo de confianza (Marina, 2010) y su mayoría de edad con respecto a quien sufre el abuso: adultos con algún parentesco -"su padrino" (Marina, 2010a p. 43) y "su tío" (Marina, 2010a p. 49)- y "amigos más grandes" (Marina, 2010a p. 51).

\subsection{Nivel primario}

La construcción de este objeto en el cuaderno de nivel primario se ubica en una relación de continuidad con lo planteado en el nivel inicial. Sin embargo, aparecen algunos nuevos significantes ubicados como componentes de este objeto. El reconocimiento y la expresión de los afectos son postulados como procesos educables cuyo objetivo consiste en el desarrollo de "capacidades emocionales" situadas en el marco del respeto por los demás (Cfr. Marina, $2009: 77)$.

El reconocimiento de los afectos es trabajado mediante la exploración de los significados atribuidos a los sentimientos de tristeza, diversión, alegría, felicidad, miedo, enojo, aburrimiento, amor, preocupación y vergüenza (Cfr. Marina, 2009 
Revista de la Escuela de Ciencias de la Educación, año 10, número 9, enero a diciembre de 2014. Páginas 65-80. ISSN 1851-6297. ISSN EN LINEA 2362-3349. EL GIRO AFECTIVO DE LA EDUCACIÓN SEXUAL. UNA LECTURA DE LOS MATERIALES didÁcticos del programa nacional de EduCación Sexual Integral. Facundo Gustavo Boccardi.

p. 80-81). La reflexión sobre estos significados establece relaciones con las variaciones que se producen de acuerdo al crecimiento de los y las educandos y con regulaciones nominadas como "el derecho a la intimidad y el respeto a la intimidad de los otros/as" (Marina, 2009 : 79). Este último punto es abordado como el establecimiento de límites por parte del propio sujeto y el respeto de los límites establecidos por los/as otros/as. En este cuaderno, los límites aparecen relacionados con dos tópicos diferentes: la vulneración de derechos y las relaciones y sentimientos amorosos. En el primero de ellos, aparece la figura de otro adulto constituido como la amenaza contra la cual se deben establecer límites para evitar la vulneración de los derechos de las niñas. Las formas de esta vulneración reciben tres nominaciones: abuso, maltrato y trata. Por otro lado, con respecto a los sentimientos y las relaciones amorosas, la figura del otro que aparece es la de un par. En este caso, se plantea como objetivo

(...) promover la generación de acuerdos sobre formas respetuosas y cariñosas de demostrar amor entre pares, límites que pueden ponerse cuando no se comparten los sentimientos, o cuando no se desea un acercamiento físico (Marina, 2009: 77).

De esta manera, la expresión afectiva entre pares aparece configurada como una práctica educable mediante la transmisión de mecanismos reguladores. Las nociones de enamoramiento y noviazgo son ubicadas en el plano de la comunicación de sentimientos íntimos y el cuidado mutuo entre un niño y una niña pertenecientes al mismo rango etario (8). En este sentido, los únicos "acercamientos físicos" entre pares nombrados en este cuaderno refieren a "besos, caricias [y] abrazos" (Marina, 2009: 77) y no aparecen como privativos de los vínculos amorosos.

\subsection{Nivel Secundario}

Tal como hemos observado, la identificación y expresión de afectos, por un lado, y la vulneración de la integridad de los sujetos, por otro, constituyen las dos grandes líneas temáticas que recorren la construcción de la afectividad como objeto discursivo en los cuadernos correspondientes a nivel inicial y a nivel primario. En el caso que nos ocupa ahora, también es posible trazar una separación relativamente equivalente entre una zona temática inherente al reconocimiento de la complejidad afectiva y otra referida al entramado de la violencia y las emociones.

Con respecto a la identificación y expresión de afectos, el enamoramiento ocupa un lugar central en estos cuadernos. Situado en el proceso de configuración de la "subjetividad joven" (Marina, 2010b: 40), aparece vinculado a signos que indican transformaciones. Las actividades didácticas propuestas trazan un abanico que recorre los "ritos de pasaje" de la niñez a la adultez en culturas no occidentales e historias de la literatura y la filmografía occidental que tematizan 
Revista de la Escuela de Ciencias de la Educación, año 10, número 9, enero a diciembre de 2014. Páginas 65-80. ISSN 1851-6297. ISSN EN LINEA 2362-3349. EL GIRO AFECTIVO DE LA EDUCACIÓN SEXUAL. UNA LECTURA DE LOS MATERIALES didÁcticos del programa nacional de Educación Sexual Integral. Facundo Gustavo Boccardi.

"el despertar del amor" y "la iniciación en la sexualidad" (Marina, 2010b : 41). La propuesta aborda la exploración de los sentimientos y las emociones comprometidos en la experimentación del "primer enamoramiento", "los cambios corporales y el despertar de la sexualidad" (Marina, 2010b: 41). Asociados a esta experimentación, aparece un conjunto de sentimientos contradictorios tales como la pasión, el amor, el sufrimiento, el disfrute, los celos, la soledad, el pudor, la crueldad y el dolor que las actividades proponen explorar. En este marco, las nociones de amor y enamoramiento se ubican de manera recurrente en relación con la noción de pareja y el inicio de las relaciones sexuales.

Con respecto al entramado que articula violencia con emociones, se puede establecer una separación entre aquellas situadas en relaciones "entre pares" y las que involucran la figura del "adulto". Ambas nociones, la de "par" y la de "adulto", son entendidas a partir de un criterio exclusivamente etario.

En cuanto a las relaciones entre pares, se nombra un conjunto de prácticas que ponen en relación la esfera de la afectividad con la violencia. Estas son: "la presión del grupo de pares", la "coerción sexual", la "violación de la intimidad o privacidad" y el "chantaje afectivo o emocional". Las dos primeras prácticas tienen como objeto las relaciones sexuales (fundamentalmente la "primera vez"); en el caso de la "presión del grupo de pares", el objetivo es el debut sexual del sujeto presionado que en la mayoría de las situaciones es designado como masculino pero no exclusivamente; en el caso de la "coerción sexual", el agente de la coerción procura mantener relaciones sexuales con el individuo destinatario de la coerción, aquí agente y destinario aparecen designados como masculino y femenino respectivamente. Con respecto a la violación de la intimidad, se mencionan prácticas de publicidad a través de las "nuevas tecnologías" que invaden la intimidad de los individuos. La actividad didáctica correspondiente a este tópico refiere la difusión de "fotos íntimas" inscripta en una relación de noviazgo heterosexual donde el varón aparece como el agente que difunde las "fotos íntimas" que tienen como referente a la mujer (Marina, 2010b: 101). A diferencia de estas prácticas configuradas en torno a la intimidad sexual entre adolescentes, el chantaje afectivo o emocional aparece como una práctica más amplia que abarca un abanico diverso de "maneras de relacionarnos" (Cfr. Bargalló et al, 2012: 31). El conjunto de estas prácticas aparece definido por el avance sobre la voluntad, el deseo y la intimidad del otro. Éstas son nominadas como interacciones injustas y dañinas, y aparecen colocadas en una relación de oposición con los "vínculos saludables" (Bargalló et al, 2012: 101).

En cuanto a las relaciones que incluyen la figura del adulto, emerge la figura del "abuso sexual". Al igual que en los manuales precedentes, el abuso sexual es definido como "cualquier clase de búsqueda y obtención de placer sexual" que tiene como agente a "una persona adulta" y como destinatario en este caso a un-a "adolescente" varón o mujer (Cfr. Marina, 2010b : 106). El 
Revista de la Escuela de Ciencias de la Educación, año 10, número 9, enero a diciembre de 2014. Páginas 65-80. ISSN 1851-6297. ISSN EN LINEA 2362-3349. EL GIRO AFECTIVO DE LA EDUCACIÓN SEXUAL. UNA LECTURA DE LOS MATERIALES didÁcticos del programa nacional de Educación SeXual Integral. Facundo Gustavo Boccardi.

abuso sexual aparece situado fundamentalmente en el "escenario familiar" y el abusador caracterizado como un adulto propio de ese espacio. En consecuencia, el "hogar" aparece configurado como un espacio de riesgo en oposición a la escuela que es configurada como un espacio signado por la protección ejercida por "adultos de confianza". Es que decir que, en esta topografía, tanto la vulneración como la protección son ejercidas por figuras adultas. Por otro lado, el abuso sexual aparece vinculado al negocio de la explotación sexual que involucra "prostitución, pornografía y trata de menores" (Marina, 2010b: 105).

\section{Conclusiones}

El recorrido por los tres niveles educativos nos permite poner de relieve la afectividad en el entramado discursivo de la Educación Sexual integral como un espacio específico de intensidad significativa. Algunas de sus modulaciones nos permitieron comprender presupuestos acerca de la educación en sexualidad que llevan las marcas de las condiciones sociales de nuestro tiempo.

La inclusión de los afectos como un componente de la educación formal repite las referencias normativas al binomio público - privado cuyo funcionamiento ocupa un lugar central en las tradiciones pedagógicas de la modernidad. Sin embargo, en este caso la funcionalidad del binomio porta marcas específicas. Aquí, conocimiento y lenguaje adquieren un estatuto equivalente definido por su operacionalidad racional que ejerce la condición de posibilidad de la comprensión y protección. Ambas nociones ocupan el lugar de los objetivos de la educación de la esfera de la afectividad trazando una articulación entre afectos, conocimiento y riesgos. En este sentido, el complejo juego que se establece entre educación pública y afectos privados articula un mecanismo regulador que funciona pautando las expresiones afectivas según parámetros diferenciados por rango etario (9).

El riesgo es, a la vez, uno de los lugares más recurrentes en la topología discursiva de la sexualidad y uno de los tropos más repelidos por la formación discursiva de los derechos humanos. En este caso, su presencia se encuentra modulada de acuerdo a los niveles educativos de los cuadernos. Con respecto a los elementos constantes, los tres niveles funcionan con la referencia a la figura adulta que aparece cargada con una doble valencia: la del abuso y la de la protección. El abuso es ubicado en la esfera privada y la protección en la esfera pública. En esta espacialización del riesgo, el conocimiento aparece como la condición de posibilidad para que la protección pública actúe sobre el abuso privado. Con respecto a las variaciones, cuando el riesgo de vulneración es ubicado en "relaciones entre pares" funciona un criterio evolutivo que asimila su intensificación al crecimiento etario. En consecuencia, su mayor presencia se encuentra en los cuadernos correspondientes al nivel secundario donde aparece ubicado principalmente en las relaciones de pareja o noviazgo. Este espacio afectivo regido por la norma de la heterosexualidad es asociado con el 
Revista de la Escuela de Ciencias de la Educación, año 10, número 9, enero a diciembre de 2014. Páginas 65-80. ISSN 1851-6297. ISSN EN LINEA 2362-3349. EL GIRO AFECTIVO DE LA EDUCACIÓN SEXUAL. UNA LECTURA DE LOS MATERIALES didÁcticos del programa nacional de Educación Sexual Integral. Facundo Gustavo Boccardi.

inicio de las relaciones sexuales y distribuye los roles inherentes a la vulneración mediante la regla de género canónica: varón vulnerador - mujer vulnerada.

A fin de cuentas, la curricularización de los afectos constituye una de las operaciones medulares de la Educación Sexual Integral. El abanico de tópicos que atraviesa este objeto discursivo ofrece indicios para explorar transformaciones y continuidades en los dispositivos que regulan la vida en el horizonte contemporáneo. Alejada de la pretensión de clausurar sentidos, la lectura propuesta se ha limitado a establecer relaciones en una superficie discursiva específica con el objeto de marcar tensiones y poner de relieve mecanismos significantes cuyas operaciones abren un campo de efectos aún poco explorado en el campo educativo argentino.

\section{Notas Bibliográficas}

(1) Graciela Morgade, María del Carmen Feijoó, Eva Giberti y Silvina Ramos.

(2) Claudia Lombardo, en representación de las iglesias evangélicas, el rabino Daniel Goldman y Myriam Andújar y María Inés Franck, por el Consejo Superior de Educación Católica -CONSUDEC.

(3) La restricción del corpus de análisis a este conjunto de cuadernos obedece a dos razones. En primer lugar, nos interesa indagar puntualmente los materiales cuyo objetivo explícito es el trabajo en las aulas y cuyxs destinatarios modelos son Ixs docentes en ejercicio. Esta restricción deja afuera del corpus otros materiales de gran interés e impacto como, por ejemplo, la revista Educación sexual integral. Para charlar en familia (2011). En segundo lugar, opera una razón de carácter eminentemente metodológico, el ángulo analítico que posibilita nuestra mirada privilegia, en esta ocasión, la materia verbal o lingüística. Esta opción deja afuera los materiales centrados en las imágenes, tales como el conjunto de láminas destinadas al trabajo en los diferentes niveles educativos. La totalidad de estas producciones se encuentra disponible en la página del Ministerio de Educación de la Nación: http://www.me.gov.ar/me_prog/esi.html

(4) Ambos cuadernos correspondientes al nivel secundario están destinados a todo el nivel y su distinción obedece a las áreas disciplinares que abarcan.

(5) En este caso, se presenta un marco conceptual general que es repetido en todos los cuadernos, mientras que las variaciones textuales no son muy extensas y refieren a las especificaciones del nivel.

(6) Si bien el discurso de la ESI le otorga a los afectos un lugar transversal que trasciende su curricularización y se encuentran presentes en las propuestas para indagar la organización de la vida institucional y abordar la relación con la comunidad educativa, en este caso nuestro enfoque se limita a su presencia en los componentes del dispositivo didáctico que analizamos.

(7) El punto inicial de los afectos desde una perspectiva evolutiva aparece colocado aún antes del nacimiento. En el apartado destinado a la gestación, se presenta una actividad que aborda los "pensamientos" y "sentimientos" de "los bebés antes, durante y después de nacer" (Marina, 2010a p. 29). Para ello, se presenta un cuento que "narra la historia de un nene dentro de la panza de su mamá" (Marina, 2010a p. 28). El cuento personifica a dicho "nene" atribuyéndole conocimientos, sensaciones y emociones. 
Revista de la Escuela de Ciencias de la Edducación, año 10, número 9, enero a diciembre de 2014. PÁginas 65-80. ISSN 1851-6297. ISSN EN LÍNEA 2362-3349. EL GIRO AFECTIVO DE LA EDUCACIÓN SEXUAL. UNA LECTURA DE LOS MATERIALES didÁcticos del programa nacional de Educación SeXual Integral. Facundo Gustavo Boccardi.

(8) En las actividades didácticas de este cuaderno se relatan tres historias de "enamoramiento", en todas se trata de un niño enamorado de una niña (Cfr. Marina, 2009 p. 24, 77 y 82$)$.

(9) Consideramos que un análisis en profundidad de esta articulación debería recorrer la genealogía de la formación discursiva de la educación sexual integral deteniéndose en la circulación de componentes provenientes del feminismo de la segunda ola que impulsan politización y publicidad de la esfera íntima con aquellos componentes de campo psi que confluyen en un modelo cultural de intimidad impregnado por los atributos de libertad e igualdad (Cfr. Squire, 2001).

\section{Referencias Bibliográficas}

- $\quad$ Angenot, M. (1982). La parole pamphlétaire. Typologie des discours modernes. París: Payot.

- Bargalló, M. L. [et al.] (2012).Educación sexual integral para la educación secundaria II: contenidos y propuestas para el aula. Buenos Aires: Ministerio de Educación de la Nación.

- Birgin, H. (1996): Derechos reproductivos en la reforma constitucional. Política y población en la Argentina: claves para el debate. Buenos Aires: Ediciones de la Flor.

- Boccardi, F. (2010). Educación sexual y perspectiva de género. Un análisis de los debates sobre la ley de educación sexual integral en la Argentina. Perspectivas de la Comunicación, Volumen 1, Tomo 1, Número 2, 48-58.

$\bullet$ (2013). Educación de la diferencia sexual. Acerca de lo que se dice que hay que enseñar a la hora de dictar educación sexual en las escuelas."Revista Punto Género No 3, 83-97.

- Bernstein, B. (1990). Clases, códigos y control II. Hacia una teoría de las transmisiones educativas. Madrid: Akal.

- Cosse, I. (2010). Pareja, sexualidad y familia en los años sesenta: una revolución discreta en Buenos Aires. Buenos Aires: Siglo XXI.

- Chopin, A. (1993). Les Manuels scolaires : histoire et actualité. Paris: Hachette.

- Esquivel, J. C. (2013). Cuestión de educación sexual: pujas y negociaciones políticoreligiosas en la Argentina democrática. Ciudad Autónoma de Buenos Aires: CLACSO.

- Felitti, K. (2009). Difundir y controlar. Iniciativas de educación sexual en los años sesenta. Revista Argentina de Estudios de Juventud, n 1, Observatorio de Jóvenes, Comunicación y Medios, Facultad de Periodismo y Comunicación Social - UNLP, Argentina.

- Foucault, M. (1987). El orden del discurso. Barcelona: Tusquests.

- Gogna, M. (2005). Estado del Arte: investigación sobre sexualidad y derechos en la Argentina: 1990 - 2002. Buenos Aires: CEDES.

- Greco, M. \& Stenner, P. (2008). Emotions: a social science reader. London: Routledge.

- $\quad$ Gregg, M. \& Seigworth, G. (2010). The Affect Theory Reader. Durham \& London: Duke University Press Books.

- Marina, M. (coord.) (2009). Educación sexual integral para la educación primaria: contenidos y propuestas para el aula. Buenos Aires: Ministerio de Educación de la Nación.

$\bullet$ (2010a). Educación sexual integral para la educación inicial: contenidos y propuestas para el aula. Buenos Aires: Ministerio de Educación de la Nación. (2010b). Educación sexual integral para la educación secundaria: contenidos y propuestas para el aula. Buenos Aires: Ministerio de Educación de la Nación. 
Revista de la Escuela de Ciencias de la Educación, año 10, número 9, enero a diciembre de 2014. Páginas 65-80. ISSN 1851-6297. ISSN EN LINEA 2362-3349. EL GIRO AFECTIVO DE LA EDUCACIÓN SEXUAL. UNA LECTURA DE LOS MATERIALES DidÁCticos del programa nacional de Educación SeXual Integral. Facundo Gustavo Boccardi.

- $\quad$ Squire, C. (2001). The public life of emotions. International Journal of Critical Psychology, Vol. 1, 27-38.

- Tosi, C. (2008). La edición de libros de texto en la Argentina. Mercado, complejidad del proceso y especificidad de saberes. Espacios de crítica y producción. Nro. 37, 70-75.

- Wainerman, C.; Di Virgilio, M. y Chami, N. (2008).La escuela y la educación sexual. Buenos Aires: Manantial. 